\title{
Le périmètre du SCOT de la région de Strasbourg
}

Jeux d'acteurs et logiques de recomposition des territoires dans une aire métropolisée

The perimeter of the SCOT of the region of Strasbourg. Stakeholders' interplay and logics of territorial recomposition in a metropolised area

Der Umkreis der SCOT der Region von Straßburg. Beteiligtenspiele und

Umstrukturierungslogik der Territorien in einer metropolisierten Umgebung

\section{Christophe Sohn}

\section{OpenEdition \\ Journals}

Édition électronique

URL : http://journals.openedition.org/rge/792

DOI : 10.4000/rge.792

ISSN : 2108-6478

\section{Éditeur}

Association des géographes de l'Est

\section{Édition imprimée}

Date de publication : 1 juin 2004

Pagination : 157-168

ISSN : 0035-3213

\section{Référence électronique}

Christophe Sohn, «Le périmètre du SCOT de la région de Strasbourg », Revue Géographique de l'Est [En ligne], vol. 44 / 3-4 | 2004, mis en ligne le 10 juin 2009, consulté le 07 septembre 2020. URL : http:// journals.openedition.org/rge/792; DOl : https://doi.org/10.4000/rge.792

Ce document a été généré automatiquement le 7 septembre 2020

Tous droits réservés 


\section{Le périmètre du SCOT de la région de Strasbourg}

Jeux d'acteurs et logiques de recomposition des territoires dans une aire métropolisée

The perimeter of the SCOT of the region of Strasbourg. Stakeholders' interplay

and logics of territorial recomposition in a metropolised area

Der Umkreis der SCOT der Region von Straßburg. Beteiligtenspiele und

Umstrukturierungslogik der Territorien in einer metropolisierten Umgebung

\section{Christophe Sohn}

\section{NOTE DE L'ÉDITEUR}

Article reçu le 25 novembre 2004, accepté le 10 janvier 2005

1 Sous l'influence conjointe des politiques de décentralisation et d'intégration européenne, de nouveaux modes d'organisation des territoires voient le jour et se combinent avec les structures existantes. Ce processus de recomposition territoriale s'inscrit dans la montée en puissance de l'intercommunalité comme maille de gestion $\mathrm{du}$ territoire. Il donne à voir de nouveaux acteurs territoriaux qui, pour exister et s'affirmer, élaborent des projets de développement et d'aménagement. Comment ces « territoires de projets » qui fleurissent au gré des initiatives locales et de l'empilement législatif des instruments de planification, de gestion et de contractualisation s'inscrivent-ils dans l'espace? Comment se positionnent-ils les uns par rapport aux autres ? Selon quelles articulations et avec quelle cohérence à la clé ?

2 Cet article se propose d'aborder un aspect particulier des recompositions à l'œuvre en se focalisant sur les schémas de cohérence territoriale (SCOT), nouvel outil de planification porteur d'un projet de territoire. Plus précisément, il s'agit d'analyser la mise en place du périmètre du SCOT de la région de Strasbourg (SCOTERS) dans le dessein d'interroger les interactions entre jeux d'acteurs et logiques de recomposition 
des territoires. Certes, le choix de s'intéresser exclusivement aux questions de périmètre peut surprendre, tant il est vrai que c'est, a priori, le projet, autrement dit les orientations en matière d'aménagement et de développement, qui donne tout son sens à l'outil. Mais si, d'une certaine manière, le mot d'ordre des acteurs territoriaux peut quelquefois se résumer à « qu'importe le flacon, pourvu qu'on ait l'ivresse ! » (Wachter et al., 2000), cette démarche se justifie toutefois dans la mesure où elle repose sur une hypothèse forte : l'établissement d'un périmètre de SCOT est porteur d'enjeux pour les acteurs qui participent à l'aménagement des espaces et à leur gestion. En filant la métaphore, on dira que la question de la taille du "flacon » et de ceux qui en ont accès est des plus importantes, puisqu'elle détermine la force de « l'ivresse » et les modalités de son partage.

3 Dans un premier temps, cette analyse va s'attacher à mettre en évidence le contexte qui a prévalu lors de la mise en place du périmètre du SCOTERS ainsi que les facteurs qui ont déterminé son tracé. La présentation des intercommunalités intégrées au schéma permettra ensuite de mieux prendre en considération le positionnement des acteurs et leurs intérêts. Enfin, l'étude des stratégies territoriales à l'œuvre dans la région de Strasbourg nous conduira à soulever la question de l'articulation des différents périmètres et de leur cohérence.

\section{La mise en place d'un nouvel outil de planification: entre nécessité et contingence}

4 Deux déterminants principaux sont à l'origine de la définition du périmètre du SCOT de la région de Strasbourg. Sur un plan institutionnel, l'opération s'inscrit dans le cadre du renouvellement de l'outillage de la planification du territoire. Au plan local, le SCOTERS participe de l'indispensable prise en compte de l'expansion de la métropole strasbourgeoise et de son aire d'attraction. Face à ces rationalités qui pèsent dans le modelage du périmètre, interviennent également des facteurs circonstanciels liés au jeu des acteurs et aux rapports de pouvoir qui sont mis en scène.

\section{A. La nécessaire adaptation des instruments de planification aux évolutions urbaines et institutionnelles}

5 Avec l'adoption, en 2000, de la loi « Solidarité et renouvellement urbain » (SRU), exit les schémas directeurs (SD) et autres plans d'occupation des sols (POS). Désormais, il convient d'évoquer les schémas de cohérence territoriale et les plans locaux d'urbanisme (PLU). Au-delà d'un simple changement de vocable, c'est en fait une vision renouvelée de la planification territoriale qui est formalisée. Dispositif phare de la nouvelle législation, le SCOT est l'outil de planification et d'orientation d'un développement qui se doit désormais d'être « durable ${ }^{1}$ » à l'échelle d'une aire urbaine ${ }^{2}$. Parfois présenté comme un anti-schéma directeur (Intercommunalités, 2001), il laisse de côté la notion de plan - déclarée obsolète - pour promouvoir l'idée de projet d'aménagement et de développement durable du territoire (le PADD). Concrètement, le nouveau schéma est là pour définir les objectifs d'aménagement que les PLU devront traduire au niveau communal. Aux orties donc l'ancienne carte de destination générale des sols qui s'est avérée, il est vrai, bien souvent trop précise, et place à une vision globale des territoires urbains et de leurs enjeux. Dans cette veine, on comprendra qu'il 
n'est plus question de juxtaposer mécaniquement des zones mono-fonctionnelles et d'appréhender de manière indépendante les différentes activités humaines qui structurent l'espace géographique. L'objectif est de concilier la maîtrise de l'étalement urbain avec l'exigence d'un développement respectueux envers les générations à venir, de promouvoir la mixité sociale et la diversité des fonctions urbaines et de tenir compte de la mobilité sans cesse croissante des personnes, tout cela dans le respect des grands équilibres entre le rural et l'urbain, entre les espaces à urbaniser et ceux à protéger (METL, 2002). Pour atteindre ces objectifs, le SCOT vise l'harmonisation des politiques sectorielles en matière de logement, de commerce, de développement économique, mais aussi de transport et d'environnement.

6 Sur le plan du périmètre, la règle retenue est simple : il se doit d'être d'un seul tenant, sans enclave et de recouvrir la totalité du territoire des établissements publics de coopération intercommunale (EPCI) compétents pour son élaboration. Au-delà de ces grands principes, la loi pose l'aire urbaine de plus de 50000 habitants comme l'échelle de référence pour l'élaboration des SCOT, soulignant ainsi l'intérêt particulier porté aux questions des déplacements pendulaires. Si le préfet est chargé de veiller au bon déroulement de la procédure, ce sont les collectivités locales qui doivent prendre l'initiative de s'unir et de négocier l'élaboration du périmètre. En somme, c'est donc le contexte local avec le jeu de ses acteurs qui va délimiter le territoire de projet, lequel pourra prendre des proportions différentes au gré des situations. On notera, pour conclure, que les exigences quant à la rationalité du périmètre rejoignent celles édictées par la loi Chevènement du 12 juillet 1999 sur la coopération intercommunale et la loi Voynet du 25 juin 1999 sur les Pays. La démarche SCOT s'insère résolument dans la dynamique de l'intercommunalité qui inscrit l'action des collectivités locales dans une logique de projet, soit au niveau intercommunal, soit à l'échelle plus vaste des Pays.

\section{B. L'irrésistible expansion de l'aire d'attraction de l'agglomération strasbourgeoise}

7 À Strasbourg, l'établissement du périmètre du SCOT trouve ses origines dans la révision du schéma directeur d'aménagement et d'urbanisme (SDAU) engagée officiellement le 20 décembre 1996 sur demande de la Communauté urbaine (CUS). En décalage avec le contexte urbain des années 90, le SDAU, adopté à la veille du premier choc pétrolier en 1973, apparaissait obsolète et incapable d'orienter les choix en matière d'urbanisation et de développement de la région. Certaines des grandes hypothèses démographiques et économiques, ainsi que les choix qui en découlaient en matière de planification urbaine, s'étaient avérés irréalistes. Ainsi, la croissance de la population résidant au sein du schéma directeur avait été largement surestimée, puisque le nombre d'habitants en 2000 avoisinait les 535000 au lieu des 708000 envisagés 25 ans plus tôt (ADEUS, 1996). Dans le même temps, la consommation d'espace avait été forte, les communes périphériques à vocation rurale subissant l'assaut de migrants pendulaires en quête d'un pavillon individuel à partir des années 70 (Limouzin, 1988 ; Schaub-Faller et Schaub, 1998). L'expansion de l'agglomération strasbourgeoise avait également contourné les prescriptions et les cadres prédéfinis pour se développer là où on ne l'attendait pas. Alors que le développement fortement structuré vers l'ouest ne s'est pas concrétisé comme prévu, l'urbanisation a filé selon un axe nord-sud. Enfin, au niveau des activités économiques, certains aménagements prévus dans le SDAU, comme la 
vaste zone à vocation pétrochimique au nord-est de Strasbourg, n'ont jamais vu le jour, en partie du fait du renversement de la conjoncture.

La révision du SDAU de 1973 demandait que soit déterminé, dans un premier temps, le périmètre dans lequel s'inscriraient les orientations fondamentales d'aménagement dans la région de Strasbourg (ADEUS, 1995). Recouvrant une aire de $780 \mathrm{~km}^{2}$ et 93 communes, le périmètre existant correspondait, à l'époque de sa définition, aux limites du secteur socio-économique dont Strasbourg était le pôle (Figure 1). Or, deux décennies plus tard, l'aire d'attraction de la métropole alsacienne ${ }^{3}$ telle que définie par l'Agence de développement et d'urbanisme de Strasbourg (ADEUS) s'est considérablement dilatée: désormais, cette dernière recouvre une zone de près de $1100 \mathrm{~km}^{2}$, soit 149 communes, pour une population de 550000 habitants. Reflet de l'expansion du périmètre d'attraction de l'agglomération strasbourgeoise, l'accroissement des migrations pendulaires, dont une majorité de déplacements domicile-travail, entre la ville-centre (Strasbourg), ses deux premières couronnes (CUS en dehors de Strasbourg) et le reste du département. Si en 1968, la CUS accueillait 24700 migrants alternants journaliers et en «émettait » 2700 , en 1990, ces chiffres sont passés respectivement à 50000 et 13900 (ADEUS, 2001).

Figure 1 : Le débordement du SDAU de 1973 par l'aire urbaine de Strasbourg.

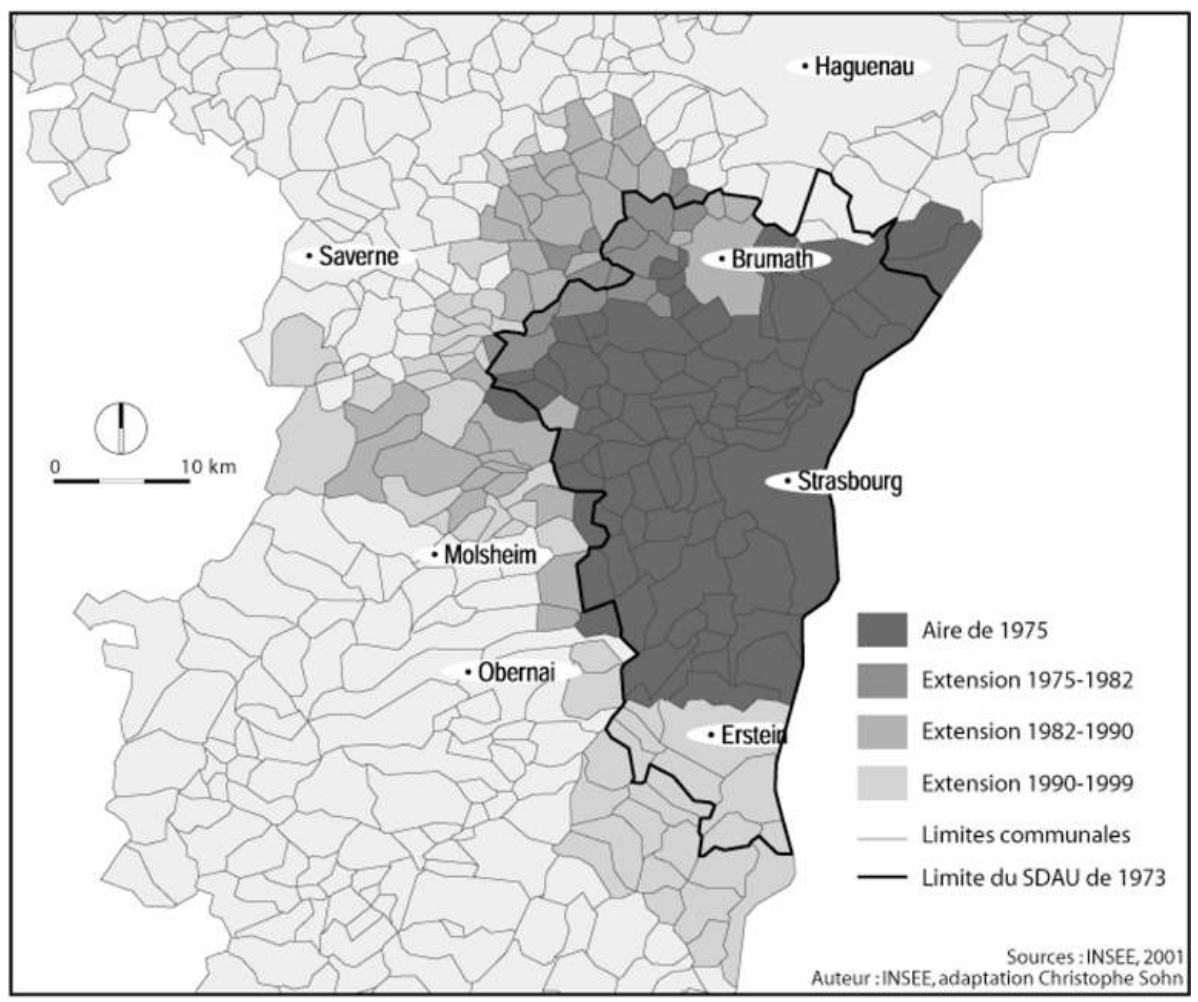

9 Cette évolution marquée par un accroissement de la zone polarisée par la capitale régionale n'est pas spécifique à Strasbourg, mais s'inscrit dans le cadre du phénomène de métropolisation qui touche la plupart des grandes villes (Ascher, 1995). Caractérisée par une concentration accrue des fonctions de commandement, des activités tertiaires et stratégiques et des emplois les plus qualifiés, la métropolisation à l'œuvre contribue à amplifier l'attractivité de l'agglomération. Cela dit, la croissance de la ville ne se fait pas aux dépens de sa périphérie. Strasbourg joue plutôt un rôle de redistribution de la 
population vers les espaces périphériques métropolisés (ADEUS, 2001). Cette diffusion de la périurbanisation dans l'ensemble de l'aire urbaine touche aussi bien les petites villes (Molsheim, Obernai, Erstein Brumath) que les bourgs-centres (Wasselonne, Benfeld, Hochfelden) ou les villages. Parallèlement à l'accroissement de leur population résidante, les communes périphériques ont également profité du dynamisme économique de la métropole à travers la diffusion des activités (ADEUS, 1998). Cette particularité de la dynamique métropolitaine à Strasbourg est liée aux spécificités de l'armature urbaine rhénane basée sur la complémentarité des villes et leur fonctionnement en réseau (Nonn, 1999). Autour de la capitale alsacienne, la région est constituée d'un maillage serré de petites villes et de bourgs-centres qui dépendent certes de la métropole, mais conservent également une capacité de structuration de leur arrière-pays rural.

\section{La définition du périmètre du SCOTERS sur la base de l'aire urbaine... ou presque}

10 Si la détermination de l'aire d'attraction de l'agglomération de Strasbourg constitue un élément important dans la définition du périmètre du SCOTERS, la prise en compte des autres aires d'aménagement $d u$ territoire et des structures de gestion s'avère indispensable. Ainsi, la présence d'anciens schémas directeurs encore en vigueur en bordure de la région de Strasbourg représente une contrainte forte, puisqu'il est extrêmement compliqué pour une commune de sortir d'un SD ou d'un SCOT pour en intégrer un autre. La géographie des diverses structures intercommunales constitue également un facteur dont il faut tenir compte ${ }^{4}$. Ce sont essentiellement les EPCI ayant compétence en matière de SCOT qui revêtent une importance stratégique, car elles peuvent choisir entre l'élaboration de leur propre schéma ou l'adhésion en bloc à un schéma portant sur une surface plus grande. Enfin, les limites informelles des espaces fonctionnels comme les bassins d'emploi et d'habitat entrent également en jeu, dans la mesure où ces entités peuvent être mobilisées dans la délimitation d'autres démarches de projet comme les chartes de Pays. Si, en définitive, l'articulation de ces différents territoires et aires géographiques n'est jamais entièrement satisfaisante, tant les logiques qui les déterminent individuellement sont différentes, l'opération de délimitation d'un périmètre est encore complexifiée du fait de la nature éminemment politique de la procédure. En effet, cette dernière repose essentiellement sur une démarche volontariste des communes et des EPCI et sur leur capacité à s'entendre. Dans le positionnement des élus locaux vis-à-vis de l'adhésion ou non de leur territoire à un SCOT, entrent également en ligne de compte les stratégies politiques des intéressés, leurs ambitions personnelles et leurs affinités avec les autres partenaires.

11 À Strasbourg, entre le moment où la procédure de révision a été lancée (20 décembre 1996) et la date à laquelle le préfet a arrêté le nouveau périmètre (30 dé- cembre 1998), des consultations et négociations entre les différentes parties prenantes eurent lieu sur la base de quatre scénarios préparés par l'Agence de développement et d'urbanisme (ADEUS). Ceux-ci variaient d'une version a minima se contentant de retoucher le périmètre du SD existant à la marge à un projet de territoire étendu recoupant peu ou prou l'aire d'attraction de l'agglomération de Strasbourg (ADEUS, 1995). Le périmètre qui fut finalement retenu recouvre en partie seulement ce que l'INSEE a identifié comme étant l'aire urbaine de Strasbourg (Figure 2). Au nord et au sud de la CUS, l'adhésion des communes s'est faite de manière raisonnée, par acceptation d'une 
logique évidente, celle d'appartenir à l'aire d'attraction de la métropole. Dans certains cas, l'extension du périmètre n'a toutefois pas été possible compte tenu de l'existence d'un schéma directeur ${ }^{5}$. Mais là où l'élargissement s'est avéré particulièrement problématique, c'est au niveau de la périphérie occidentale de l'aire urbaine. Compte tenu de la non-adhésion au SCOTERS de centres urbains secondaires pourtant clairement situés dans l'aire d'attraction de l'agglomération strasbourgeoise comme Wasselonne, Molsheim ou encore Obernai, c'est finalement la pertinence du périmètre qui s'en trouve diminuée. Revenons sur les raisons qui ont amené certains territoires périphériques à rester en-dehors du SCOTERS.

Figure 2 : Aires urbaines et périmètres d'aménagement dans le Bas-Rhin en 1999.

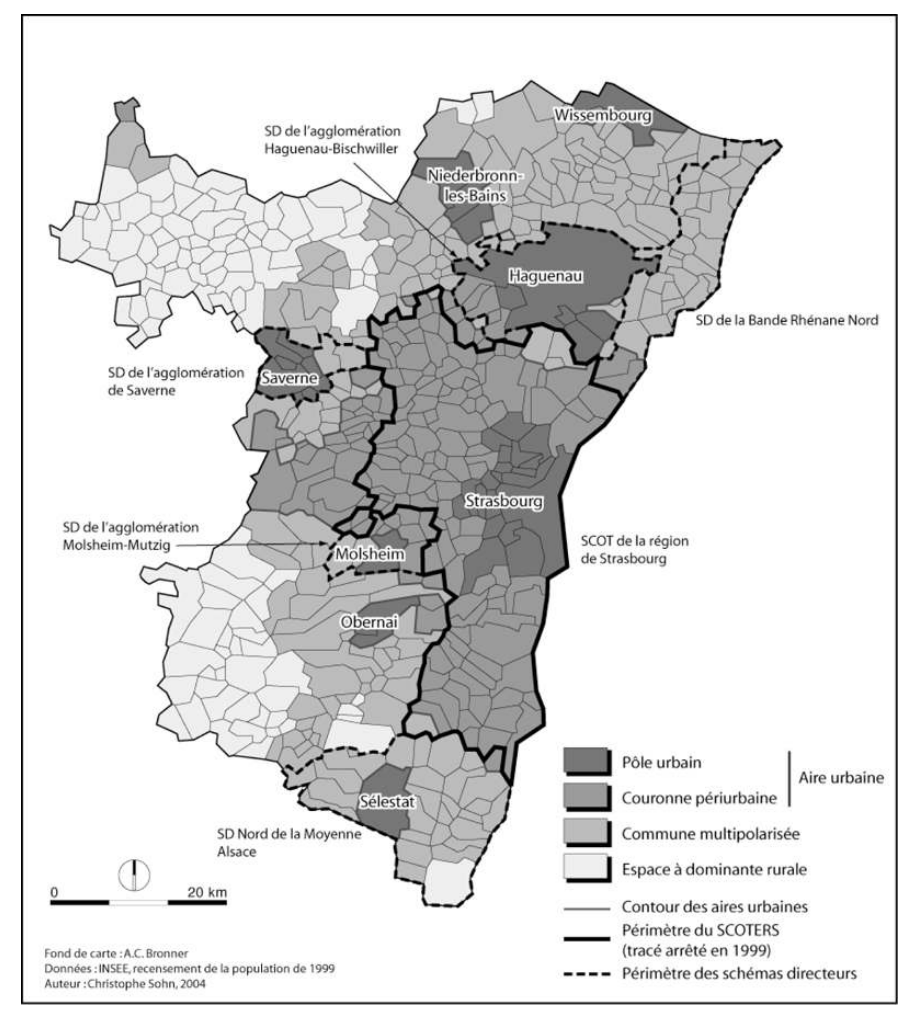

Pour Wasselonne, il s'agit avant tout d'un conflit de personnes opposant le maire de la commune avec celui de Marlenheim, deux centres urbains proches et concurrents. En effet, à partir du moment le second avait accepté d'intégrer le périmètre du futur SCOT, le premier ne pouvait que refuser une telle proposition. Loin de toute considération portant sur l'aménagement et le développement de leur territoire, on se situe là dans le registre médiocre de la guerre de clochers et de l'opposition systématique des deux élus qui y sont engagés. Le cas de Molsheim s'avère plus complexe, dans la mesure où les raisons qui ont motivé le refus sont à peu près aussi pertinentes que celles qui plaidaient en faveur d'une adhésion. Compte tenu de la proximité géographique de Molsheim avec la CUS et de l'importance des migrations alternantes entre la petite ville du piémont et l'agglomération strasbourgeoise ${ }^{6}$, il aurait été souhaitable que le périmètre du futur SCOTERS absorbe celui du schéma directeur de Molsheim-Mutzig. En dépit de la complexité de la procédure, l'intérêt de l'opération aurait été pleinement justifié. C'était pourtant sans compter sur le refus déterminé des élus de Molsheim et des onze communes intégrées au sein du SD. Outre la personnalité du maire et son opposition à l'égard de Strasbourg, l'existence d'une véritable identité territoriale 
basée sur des racines anciennes d'autonomie urbaine très ancrées en Alsace, le souci de rester maître de ses finances locales (particulièrement la taxe professionnelle) et de préserver une certaine autonomie de décision vis-à-vis d'une CUS perçue comme «phagocytant» sa périphérie comptent parmi les raisons qui expliquent le refus. Bien sûr, le discours sur la perte d'autonomie est bercé d'illusions, mais il s'avère mobilisateur pour galvaniser l'opposition classique des communes favorisées à la métropole proche. Précisons qu'on retrouve grosso modo les mêmes motivations et des représentations similaires auprès des communes d'Obernai et de Rosheim.

13 À côté de ces considérations franco-françaises, l'établissement du périmètre du SCOTERS a pris en considération la dimension transfrontalière des dynamiques métropolitaines, poursuivant ainsi la démarche entreprise en 1990 lors de l'élaboration $\mathrm{du}$ projet d'agglomération Strasbourg-Kehl. Si, en tant qu'outil de planification "nationale", le SCOTERS ne pouvait étendre son périmètre au-delà du territoire français, la procédure de révision de l'ancien schéma directeur a été appréhendée dans un cadre transfrontalier. Le territoire retenu pour réaliser les études préalables a ainsi été étendu au Kreis ${ }^{7}$ de l'Ortenau qui apparaît comme le pendant allemand de la région de Strasbourg (ADEUS, 2001). En dépit des différences de conception des documents d'urbanisme et d'aménagement français et allemand et des décalages dans l'échéancier des révisions, l'objectif visé par ce projet Interreg III était de mieux définir les enjeux et les grandes orientations servant de base à l'élaboration des documents de planification entre les deux pays et de tendre, à terme, vers une mise en cohérence des projets d'aménagement de part et d'autre du Rhin. Mais pour cela, encore faut-il que l'enjeu transfrontalier ne soit pas oblitéré par les divergences d'intérêts entre l'agglomération strasbourgeoise et sa périphérie (Reitel, 2000).

\section{De la diversité des territoires intégrés au SCOTERS a la divergence de leurs intérêts}

14 Aborder la question des relations entre les territoires de gestion au sein du SCOTERS nécessite de préciser quels types d'acteurs sont en scène. Si l'échelon communal tient encore une place de choix dans l'édifice territorial français, c'est à l'échelle des regroupements de communes qu'apparaissent les acteurs clés du nouveau territoire de projet en cours d'élaboration. Avant de considérer les caractéristiques des territoires en présence et la nature de leurs relations, intéressons-nous à la dynamique de l'intercommunalité à l'œuvre dans la région de Strasbourg et son incidence sur la définition du périmètre du SCOTERS.

\section{A. Le rôle actif de l'intercommunalité dans l'établissement du périmètre du SCOTERS}

Lors des travaux préliminaires menés au milieu des années 90 en vue de réviser le SDAU, les structures intercommunales établies dans la région présentaient encore une grande diversité de statuts et de compétences. À côté des établissements à fiscalité propre (communauté de communes, district) subsistaient des structures de gestion de type SIVU / SIVOM ${ }^{8}$. Cette hétérogénéité des EPCI était certes le résultat de l'accumulation de différentes politiques visant à encourager la coopération intercommunale, mais aussi d'un engouement contrasté des élus locaux pour cette 
question. En 1999, avec l'entrée en vigueur de la loi Chevènement visant à simplifier l'organisation territoriale française et à renforcer la coopération intercommunale, les diverses structures héritées ont progressivement été transformées en communautés de communes. Dans le même temps, on a assisté à de nouveaux regroupements intercommunaux, et donc à la diminution du nombre de communes isolées. Il faut dire que certaines collectivités locales de la périphérie de Strasbourg - comme les communes à vocation agricole du Kochersberg - avaient, pendant longtemps, tourné le dos aux formes de coopération intercommunale existantes, se privant par là même d'un outil potentiellement efficace pour contrôler l'expansion de la ville-centre (Limouzin, 1999).

Cette montée en puissance de l'intercommunalité dans la région de Strasbourg a joué un rôle clé dans la stabilisation du périmètre du SCOTERS. En attendant l'élaboration d'un projet d'aménagement et de développement durable du territoire porteur d'une vision commune et susceptible de fédérer les identités, c'est l'appartenance des collectivités locales à des communautés de communes qui consolide le territoire en devenir. À partir du moment où les communes ont délégué leur compétence en matière d'aménagement de l'espace - et donc en matière de SCOT - à la structure intercommunale qui les chapeaute, c'est cette dernière qui prend la décision d'intégrer le SCOT ou pas. Et dans le cas où une commune souhaiterait quitter le schéma, elle devrait d'abord sortir de l'EPCI auquel elle a délégué certaines de ses compétences gestionnaires. Ce sont donc bien les établissements de coopération intercommunale qui, dans une large mesure, ont modelé le périmètre du SCOTERS en déterminant l'intégration des communes qu'ils regroupent.

La situation diffère pour les communes isolées, dans la mesure où elles apparaissent plus libres de leurs choix. Dans le cas du SCOTERS, cette marge de manœuvre s'est traduite par une modification du périmètre au niveau du front entre la CUS et Molsheim en 2003. Dans un premier temps, les communes isolées de Duppigheim et de Duttlenheim (Figure 3) avaient été intégrées au SCOTERS. Leur dépendance économique et fonctionnelle vis-à-vis de l'agglomération de Strasbourg avait motivé cette décision. C'était sans compter avec la volonté de la communauté de communes de MolzheimMutzig d'élargir son emprise en direction de la métropole afin de s'affirmer face à une CUS soupçonnée de vouloir "cannibaliser " sa périphérie à l'aide du SCOTERS. Ainsi, lorsqu'à la suite des sollicitations de Molsheim, les deux communes acceptèrent d'intégrer sa communauté de communes, elles durent se résigner à quitter le giron du SCOTERS. Dans cette nouvelle union, la mariée n'arrivait pas les mains vides puisque Duttlenheim accueille, avec deux autres communes, une des grandes zones d'activités économiques du Bas-Rhin, le Parc d'activités de la Plaine de la Bruche. La présence de nombreuses entreprises sur le ban de la commune avait déjà constitué un des enjeux principaux lors de son intégration dans le SCOTERS, dans la mesure où pour la CUS, Duttlenheim profite de la proximité de l'agglomération strasbourgeoise dans le cadre de la zone d'activités économiques en récoltant la taxe professionnelle. Il lui apparaissait donc logique que la commune coopère avec l'agglomération strasbourgeoise et participe à l'accompagnement humain et social de la main-d'œuvre liée à ces entreprises, cela d'autant plus que la zone avait été planifiée dans le SDAU de 1973. Si, en définitive, le retrait de ces communes était inévitable du fait de leur volonté d'intégrer une structure intercommunale extérieure au SCOTERS, cette valse-hésitation illustre les tiraillements de territoires de marges soumis aux logiques et aux pressions 
contradictoires entre la métropole et les centres urbains secondaires qui gravitent dans sa périphérie.

Figure 3 : Les structures intercommunales à fiscalité propre dans le Bas-Rhin en 2004.

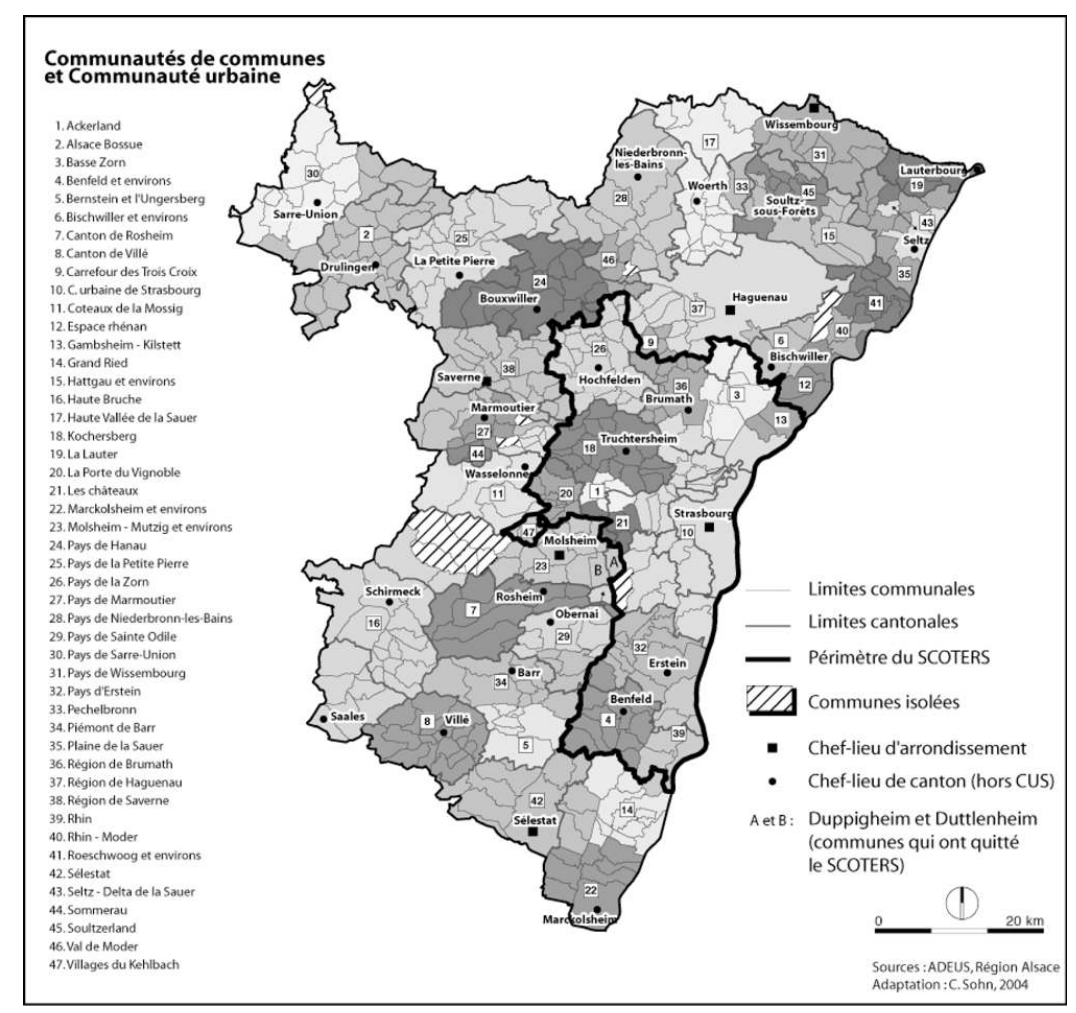

\section{B. Des territoires institutionnels de poids variables et de compétences inégales}

Le périmètre du SCOTERS regroupe finalement 139 communes comptant au total près de 575000 habitants. Sur ces 139 communes, 27 sont intégrées à la Communauté urbaine de Strasbourg et 111 sont regroupées au sein de douze communautés de communes, la commune de Blaesheim ne faisant partie d'aucune structure intercommunale (Figure 3). À elle seule, la CUS renferme 450000 habitants, soit près de $80 \%$ de la population résidant dans l'aire du SCOTERS (INSEE, 1999). Sur le plan des bases économiques, on retrouve peu ou prou les mêmes déséquilibres entre l'agglomération de Strasbourg et les communes périurbaines, la première concentrant la majorité des activités et des emplois. À cette différenciation par le poids démographique et économique des territoires intercommunaux, il faut ajouter de fortes disparités en matière de compétence gestionnaire et de ressources financières.

La CUS a été créée de manière autoritaire par la loi du 31 décembre 1966 en même temps que trois autres communautés urbaines dans le cadre de la politique d'aménagement du territoire basée sur la notion de métropole d'équilibre. En encadrant le développement de Strasbourg au-delà de ses limites communales, l'objectif affiché par l'État était de donner les moyens à la capitale alsacienne de remplir son rôle de métropole régionale et de faire contrepoids à l'agglomération 
parisienne. Progressivement, les capacités d'action de la CUS se sont accrues : outre la constitution d'un véritable territoire intercommunal au sens politique du terme, la Communauté urbaine s'est dotée d'une organisation technique et administrative originale $^{9}$ et de compétences gestionnaires élargies. Face à ce territoire fortement intégré et doté de solides ressources, les communautés de communes de la périphérie présentent des situations fort diversifiées. Au nord et au sud de la CUS, les EPCI forment des ensembles relativement importants. Ces structures se sont constituées autour de petites villes et de bourgs-centres qui ont conservé un rôle de centralité sur leur arrière-pays. En revanche, dans la partie ouest de la région de Strasbourg, les regroupements communaux opérés principalement sur des bases affinitaires ont engendré un morcellement des territoires de coopération intercommunale (Limouzin, 1999). Le cas extrême est certainement celui des Villages du Kehlbach, une communauté de communes d'à peine 1500 habitants répartis sur trois communes et qui s'est constituée en réaction à la mésentente des maires de Marlenheim et de Wasselonne au sujet de la création d'une grande structure intercommunale sur tout le canton.

Regroupés au sein d'un Syndicat Mixte chargé d'élaborer le SCOTERS, les enjeux de ces différentes collectivités sont susceptibles de diverger. Pour la CUS qui préside le Syndicat et détient la moitié des sièges du Comité syndical ${ }^{10}$, il s'agit avant tout de fédérer les territoires périurbains situés dans son aire d'attraction de manière à conforter sa position de métropole, tant à l'échelle européenne qu'au niveau de la région urbaine. Les logiques de développement majeures sont celles d'une dynamique métropolitaine «multicarte» (Nonn, 1998) qui, tout en donnant la part belle à "l'ambition européenne» de Strasbourg (Kleinschmager, 1997), n'omet pas l'aire métropolisée. Le problème est que cette dynamique est de nature à susciter une certaine méfiance auprès des territoires périphériques qui craignent d'être phagocytés par un centre hégémonique. Rappelons que lors des débats préliminaires portant sur l'élaboration du SCOTERS, la méfiance s'est traduite à l'occasion par de la défiance, l'outil de planification ayant été perçu par certains comme un instrument de contrôle du développement des territoires périurbains aux mains de la CUS, voire comme un moyen pour cette dernière d'étendre son périmètre. Les communes périphériques qui ont finalement choisi d'intégrer le SCOTERS se retrouvent pour la plupart confrontées à un double enjeu : profiter du dynamisme de la métropole tout en évitant la déferlante résidentielle, donc de migrants pendulaires qui, à terme, risquent de transformer leur localité en cité-dortoir. Parce qu'il n'est pas évident que la participation des territoires périurbains au SCOTERS leur permette de faire valoir leurs intérêts au même niveau d'importance que ceux portés par la CUS, laquelle mène les débats au sein du Syndicat Mixte et possède une capacité d'ingénierie propre autrement puissante, des stratégies d'autonomisation ont été entreprises par certains d'entre eux.

\section{Des stratégies d'acteurs qui instrumentalisent les recompositions territoriales}

21 La mise en place du SCOT de la région de Strasbourg intervient dans une période de forte recomposition des territoires institutionnels. Outre la dynamique de l'intercommunalité déjà évoquée, il faut souligner l'emballement actuel de la part des acteurs locaux et des collectivités territoriales pour la création de territoires de projet 
tels que les Pays. Comme certains des territoires participant au SCOTERS adhèrent également à ces démarches de projets, les nouveaux périmètres qui émergent doivent être pris en compte dans l'analyse des stratégies d'acteurs.

\section{A. Le double jeu des territoires charnières en quête d'autonomisation}

Dans le dessein de relativiser leur dépendance vis-à-vis de Strasbourg et de sa Communauté urbaine, la plupart des groupements intercommunaux périurbains ont entrepris de s'associer à des territoires extérieurs au SCOTERS dans le cadre de l'élaboration de projets de territoire. Sans remettre en cause leur adhésion au SCOTERS, ces EPCI sont en quête de marges de manœuvre et d'autonomie locale. Les stratégies d'alliance nouées trouvent une expression privilégiée dans les démarches de constitution de Pays, qui, en l'espace de quelques années, ont été adoptées par pratiquement toutes les communes de la région. Les Pays dont il est question ici sont issus de la loi Voynet de 1999. Il s'agit de territoires de projet qui associent élus locaux, associations, chefs d'entreprises, responsables syndicaux et citoyens dans une approche partenariale de développement local (La Gazette, 2004). Espace de débat, le Pays a pour vocation de porter un projet et de se prêter aux contrats de plan Etat-Région. Si SCOT et Pays se différencient sur bon nombre de points et ont plutôt vocation à être complémentaires, les deux outils se rejoignent néanmoins sur la question de l'élaboration d'un projet de territoire.

Dans le giron du SCOTERS, pratiquement toutes les communautés de communes participent à ce type de projet, certaines faisant d'ores et déjà partie d'un Pays reconnu (Figure 4). Il faut dire qu'en Alsace la Région joue un rôle actif dans la promotion de ces structures, une manière pour cette institution d'être présente dans le débat sur l'aménagement des territoires. Seules les communautés de communes du Kochersberg, de l'Ackerland et des Châteaux, fortement dépendantes de la CUS sur un plan économique, n'ont pour l'instant pas entamé de démarche visant l'adhésion à un Pays. Précisons qu'il s'agit de territoires limitrophes de la CUS, où les bourgs peinent à jouer un rôle de lieu de centralité et où une majorité de résidants travaille dans l'agglomération (Fausser, 2002). Pour les territoires périphériques structurés autour de pôles secondaires comme Erstein ou Brumath et désireux de conserver une certaine autonomie de décision vis-à-vis de la CUS, la participation à un Pays constitue une aubaine (Fausser, 2002). En effet, la situation de territoire charnière entre le SCOTERS et un Pays structuré autour d'une ville moyenne comme Sélestat ou Haguenau les place dans une position privilégiée pour dialoguer avec des acteurs importants et jouer le rôle de relais. Précisons toutefois que, dans la mesure où la stratégie de la double appartenance leur permet d'abord de relativiser leur dépendance vis-à-vis de la métropole, cette démarche semble avant tout motivée par une logique d'opportunité (Habay, 2003). 
Figure 4 : L'adhésion des collectivités périphériques du SCOTERS aux Pays environnants (BasRhin).

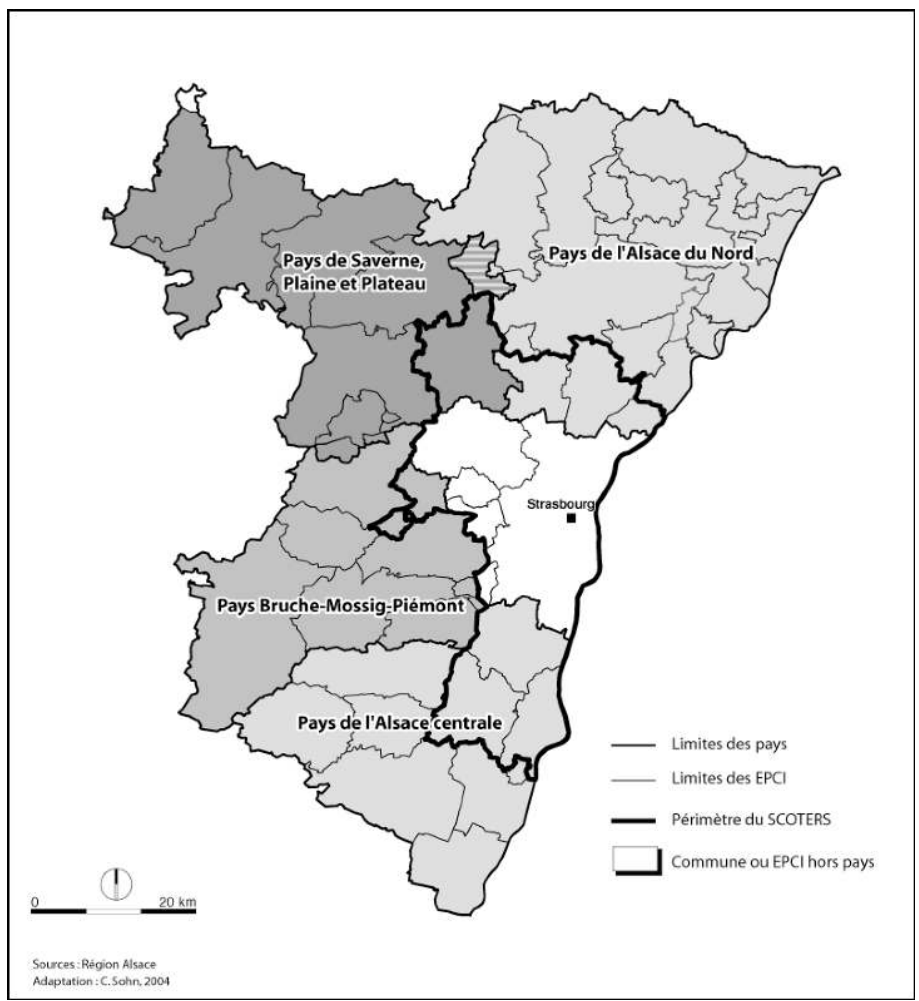

24 L'autre forme d'ouverture qui a été expérimentée réside dans la coopération transfrontalière, comme le suggère Vis-à-vis, un groupement local de coopération transfrontalière ${ }^{11}$ constitué autour d'Erstein et Lahr. Associant quatre communautés de communes françaises et sept communes allemandes, ce groupement transfrontalier s'est fixé pour objectif de promouvoir des études en vue de la construction d'un pont sur le Rhin, de favoriser les liaisons de transports publics transfrontalières et d'organiser des manifestations culturelles et sportives.

\section{B. L'ambiguîté des stratégies à géométrie variable de la métropole}

Compte tenu de la méfiance des communes périurbaines, l'objectif de la CUS est d'œuvrer à l'émergence d'une communauté de destin au sein du périmètre du SCOTERS afin de renforcer la cohésion des territoires. Ainsi, c'est pour limiter les risques de fragmentation territoriale que la mise en place d'une échelle de planification intermédiaire entre celle de la communauté de communes et celle du SCOTERS (les schémas de secteur) n'a jusqu'à présent pas été entreprise, et ce malgré les initiatives de certains acteurs institutionnels comme la Région Alsace. L'intérêt d'une telle démarche parait pourtant évident, dans la mesure où elle permettrait une meilleure prise en compte des problématiques spécifiques de chaque territoire et, à terme, la consolidation de l'espace du SCOTERS comme échelon de solidarité territoriale et fiscale entre l'agglomération et sa périphérie. Ce peu d'enthousiasme à l'égard de la question de la territorialisation des projets à une échelle géographique plus fine que celle du SCOTERS s'accompagne de l'absence d'outils de contractualisation territoriale au sein du périmètre. En ce qui concerne l'établissement d'un contrat d'agglomération au niveau de la CUS, les actuels responsables politiques issus des élections de mars 2001 
n'ont, semble-t-il, pas voulu reprendre à leur compte le projet d'agglomération qui avait été élaboré en 2000 par l'équipe municipale précédente (CUS, 2000). Quant à la création d'un Pays, cette démarche n'a pas été envisagée, dans la mesure où ce type de projet apparaît redondant avec celui porté par le SCOT aux yeux des responsables du Syndicat Mixte (Grossmann et Reverdy, non daté). En fait, le plaidoyer des dirigeants de la CUS porte sur la création d'un seul outil de planification et de contractualisation, c'est-à-dire une sorte de fusion entre SCOT et Pays. Au-delà des arguties sur les caractéristiques institutionnelles des outils et de leur fonction réelle ou supposée, ne pas œuvrer à la constitution d'un Pays centré sur l'agglomération strasbourgeoise, c'est préserver la prééminence du SCOTERS comme territoire de projet et la domination de la Communauté urbaine comme organe de gestion et de décision. Ce faisant, on élude la question du portage institutionnel des projets retenus, dans la mesure où le Syndicat Mixte constitué pour piloter l'élaboration du SCOT n'a pas vocation à les gérer ${ }^{12}$. Notons, au passage, que l'on restreint également la participation de la société civile et de ses différents acteurs aux débats sur les orientations à retenir, dans la mesure où, dans ce domaine, la procédure d'établissement d'un SCOT apparaît bien plus verrouillée que celle conduisant à la création d'un Pays. En effet, la nature institutionnelle de la démarche SCOT ne laisse que peu de place à la société civile, aux associations ou aux groupes de pressions.

À l'échelle de l'aire urbaine, se pose la question de l'interdépendance du SCOTERS avec les SCOT voisins dont l'élaboration est soutenue par le Conseil général du Bas-Rhin ${ }^{13}$. Dans la mesure où le SCOTERS ne recouvre pas l'ensemble de l'aire d'attraction de l'agglomération strasbourgeoise, la cohérence des orientations retenues dans les différents schémas à l'étude constitue un enjeu de taille ${ }^{14}$. S'il apparaît évident que l'aménagement et le développement de la région de Stras- bourg doit s'appréhender en intégrant les secteurs de Molsheim (SD de Molsheim-Mutzig et environs) et ObernaiBarr-Rosheim (SCOT du Piémont des Vosges), reste posée la question de la collaboration entre l'agglomération strasbourgeoise et les centres urbains situés en dehors du SCOTERS, mais qui participent de fait aux dynamiques économiques, sociales et territoriales de son aire urbaine. Le fait que la réalisation des études préalables à l'établissement du projet d'aménagement et de développement durable (PADD) ait tenu compte de la région allemande de l'Ortenau tout en ignorant le bassin de Molsheim en dit long sur l'état des collaborations entre les villes voisines et l'agglomération strasbourgeoise.

N'allons toutefois pas en déduire hâtivement que la coopération transfrontalière en matière de planification territoriale a définitivement vaincu les obstacles qui se dressent devant un tel exercice ni que Strasbourg aurait réussi du côté allemand ce qu'elle n'a, pour l'instant, pas été en mesure de réaliser sur le territoire français. Si la collaboration entre les deux rives du Rhin est allée bon train dans le cadre du projet de réalisation d'un Livre Blanc, force est de constater que la réalisation des projets transfrontaliers retenus n'est plus à l'ordre du jour (ADEUS, 2004). En outre, la relance en janvier 2003 du projet de création d'un "Eurodistrict» est venue brouiller la lisibilité du périmètre de coopération transfrontalière retenu jusqu'alors. Dans un premier temps, les chefs d'État français et allemand avaient annoncé la création d'un futur Eurodistrict «Strasbourg-Kehl ». Il est cependant vite apparu qu'un tel périmètre ne saurait être satisfaisant, ne serait-ce que par rapport au déséquilibre démographique existant entre les deux collectivités (264 000 habitants pour la ville de 
Strasbourg et 33000 pour celle de Kehl). Pour réduire la dissymétrie, le périmètre a été élargi à la CUS, côté français, et à la circonscription de l'Ortenau, côté allemand, soit respectivement 455000 et 400000 habitants en 1999. La délimitation de l'Eurodistrict sur la base de la CUS et non pas du SCOTERS est justifiée par les responsables de l'agglomération strasbourgeoise, dans la mesure où la première est une collectivité dotée d'une assemblée délibérante et d'un exécutif, ce que n'est pas le SCOTERS. Il faut dire que le projet des élus français et allemands est de dépasser le cadre d'un simple groupement local de coopération transfrontalière pour réaliser une véritable "communauté administrative transfrontalière ", l'ambition ultime étant de doter l'agglomération bi-nationale d'un statut européen (MOT, 2003). La réalisation de ce projet passera vraisemblablement par un renforcement de l'autonomie de la Communauté urbaine de Strasbourg à l'égard des collectivités territoriales alsaciennes, mais aussi de l'État (Reitel, 2000). D'où la crainte de certains élus des communes périphériques de voir le périmètre de l'Eurodistrict renforcer le clivage entre la CUS d'un côté et son arrière-pays de l'autre. Preuve d'une certaine incohérence dans la définition du périmètre de l'Eurodistrict: le fait qu'il ait été annoncé qu'à terme, ce dernier pourrait être étendu à l'ensemble des structures intercommunales du SCOTERS (Lenoir et Bury, 2003).

En définitive, force est de constater que l'agglomération de Strasbourg et les territoires périurbains, bien que regroupés au sein d'un même périmètre de SCOT, poursuivent des stratégies territoriales divergentes. La première est engagée dans un projet d'Eurodistrict qui, pour l'instant, exclut les espaces périphériques de la métropole côté français, ce qui apparaît contradictoire avec ses objectifs d'intégration affichés par ailleurs. Les seconds recherchent une certaine autonomie en intégrant des démarches de Pays qui ne recouvrent que partiellement l'aire du SCOTERS et ne s'articulent pas avec le pôle métropolitain. Si le défi auquel font face les acteurs participant au schéma de cohérence territoriale est bien de contrer les « forces centrifuges » à l'œuvre, l'enjeu majeur réside dans l'articulation des différents périmètres et la mise en cohérence des projets dont ils sont porteurs.

\section{Conclusion}

L'enchevêtrement des limites et le recouvrement partiel des territoires de projet au sein de l'aire urbaine de Strasbourg pose la question plus générale du fonctionnement des systèmes territoriaux ainsi structurés. Dans une certaine mesure, la multiplication des périmètres a quelques avantages, en théorie du moins. En superposant des territoires de projet qui ne se recouvrent que partiellement, on tend à relativiser l'impact de leur frontière respective. À la fois dedans et dehors, les territoires périphériques sont à la croisée des informations. En lieu et place d'une intégration exclusive, apparaissent des situations d'entre-deux mieux à même d'appréhender la complexité des dynamiques territoriales à l'œuvre, d'autant qu'il ne saurait exister de cadre englobant unique à la régulation de tous les enjeux (Vanier, 2002). Dans cette logique de la "variété requise ${ }^{15}$ » qui donne la part belle aux appartenances multiples et relatives, émergent des possibilités de jeu et d'articulation entre les différentes échelles des dynamiques territoriales et urbaines, que ce soit au niveau intercommunal, régional (inter-SCOT) ou transfrontalier. Cet enchevêtrement n'est toutefois pas sans risque. La multiplication des territoires de projet tend à dupliquer les discussions, à 
alourdir les procédures et à obscurcir le fonctionnement d'un dispositif institutionnel déjà fort complexe. Par ailleurs, l'interterritorialité n'est pas toujours motivée par le souci de mieux gérer, de donner du sens aux politiques publiques ou de prendre en compte les pratiques et les besoins des populations; elle peut parfois résulter de l'instrumentalisation des outils par des collectivités qui cherchent à renforcer leur pouvoir ou à légitimer leur action. La multiplication des territoires ou leur émiettement pose donc, en définitive, la question du sens de ces initiatives et de la cohérence des orientations retenues par les différents acteurs responsables, surtout en l'absence de régulation efficace. En reprenant les bons mots de la maxime d'Alfred de Musset citée en introduction, la multiplication des «flacons » et les mélanges plus ou moins raisonnés auxquels ils donnent lieu, ne tendent-ils pas à faire passer ceux qui en abusent, de l'ivresse à la nausée?

\section{BIBLIOGRAPHIE}

ADEUS (1995). - Quel périmètre pour le futur schéma directeur de la région strasbourgeoise?, Agence de Développement et d'Urbanisme de l'Agglomération Strasbour- geoise, Strasbourg, 84 p.

ADEUS (1996). - Atlas de la région de Strasbourg, Agence de Développement et d'Urbanisme de l'Agglomération Strasbourgeoise / La Nuée Bleue, 262 p.

ADEUS (1998). - Profil 1997 des Zones d'Activités du Bas-Rhin, analyse thématique et géographique, ADEUS - Cg67, $38 \mathrm{p}$.

ADEUS (2001). - Premier bilan de l'état des lieux, Agence de Développement et d'Urbanisme de l'Agglomération Strasbourgeoise, $21 \mathrm{p}$.

ADEUS (2004). - Livre Blanc de la région transfrontalière Strasbourg-Ortenau, Agence de Développement et d'Urbanisme de l'Agglomération Strasbourgeoise, 158 p.

ADEUS (2004). - Aménagement du territoire, La coopération transfrontalière, Dimension Villes, Agence de Développement et d'Urbanisme de l'Agglomération Strasbourgeoise, mars, $n^{\circ} 43$.

ASCHER F. (1995). - Métapolis ou l'avenir des villes, Paris, Editions Odile Jacob, 347 p.

BONIN N. (2003). - La ZAC intercommunale du Pays d'Erstein : un outil d'affirmation économique et de positionnement territorial face à la Communauté urbaine de Strasbourg, Mémoire de maîtrise d'aménagement, Université Louis Pasteur, Strasbourg, 136 p. + annexes.

COMMUNAUTÉ URBAINE DE STRASBOURG (2000). - 2e Projet d'agglomération 2000-2010, Strasbourg, CUS.

FAUSSER R (2002). - Les « frontières » de la Communauté urbaine de Strasbourg : les relations entre territoire métropolitain et territoires métropolisés, le passage des limites aux frontières, Mémoire de maîtrise de géographie humaine, Université Louis Pasteur, Strasbourg, 163 p.

GROSSMANN R., REVERDY M. (s.d.). - Scot et Pays : vers un outil unique ?, http://www.cfdu.org.

HABAY D. (2003). - Le Pays d'Erstein, un espace charnière ?, Mémoire de maîtrise de géographie humaine, Université Louis Pasteur, Strasbourg, 138 p. + annexes. 
INSEE (1999). - Quantiles Alsace, $n^{\circ} 68$, juillet.

INSEE (2001). - « L'art de découper l'espace : les nouvelles aires urbaines », Chiffres pour l'Alsace, Revue $\mathrm{n}^{\circ} 2$, juin, $7 \mathrm{p}$.

INTERCOMMUNALITÉS (2001). — «SCOT : le bon outil ne fait pas le bon ouvrier », ADCF, http:// www.intercommunalites.com/ communautes/document1454.htm

KLEINSCHMAGER R. (1997). - Strasbourg, une ambition européenne, Paris, Anthropos-Economica, 216 p. LA GAZETTE DES COMMUNES (2004). - « Créés il y a dix ans, les pays ont-ils trouvé leur place? », Dossier, 13 septembre.

LENOIR N., BURY H. M. (2003). - Eurodistrict Strasbourg-Kehl, Déclaration commune de la Ministre déléguée aux affaires européennes, Mme Noëlle Lenoir, et du Ministre délégué aux affaires européennes de la république fédérale d'Allemagne, M. Hans Martin Bury, Strasbourg, le 30 juin, http://www.diplomatie.gouv.fr/actu/

LIMOUZIN P., dir. (1988). - Les mutations spatiales dans l'agglomération de Strasbourg et sa périphérie, Association des publications près des universités de Strasbourg, Strasbourg, $250 \mathrm{p}$.

LIMOUZIN P. (1999). - « L'intercommunalité en Alsace : l'exemple du Bas-Rhin », Mosella, tome 24, $\mathrm{n}^{\circ}$ 1-2, mai, p. 248-253.

MÉLÈSE J. (1968). - La gestion par les systèmes. Essai de praxéologie, Paris, Ed. Hommes et Techniques. MINISTÈRE DE L'EQUIPEMENT, DES TRANSPORTS ET DU LOGEMENT, METL (2002). — « La mise en place des SCOT : premières tendances ", Espaces, $\mathrm{n}^{\circ}$ 14, Lettre d'information bimestrielle de la Direction Générale de l'Urbanisme, de l'Habitat et de la Construction, http://

www.logement.equipement.gouv.fr/

MOT (2003). - « Les agglomérations transfrontalières », Les cahiers de la MOT, n 3, Mission opérationnelle transfrontalière, $20 \mathrm{p}$.

NONN H. (1998). - « Une approche des fonctions internationales des métropoles : activités et acteurs à Strasbourg ». In : REYMOND H., CAUVIN C., KLEINSCHMAGER R., coord., L'espace géographique des villes, Paris, Anthropos-Economica, p. 473-505.

NONN H. (1999). - Villes et aménagement régional en Alsace, Paris, La Documentation française, 246 p. REITEL B. (2000). - « La frontière, un enjeu dans la construction de l'identité des agglomérations transfrontalières (agglomération trinationale de Bâle ; Strasbourg-Kehl) ». In : FOURNY-KOBER M.-C., dir., Le rapport à la frontière et la construction d'espaces transfrontaliers, Rapport d'étude pour la DATAR, Grenoble, 6 volumes, 2003.

SCHAUB-FALLER M., SCHAUB G. (1998). - « Coalescence et lotissements ». In : REYMOND H., CAUVIN C., KLEINSCHMAGER R., coord., L'espace géographique des villes, Paris, Anthropos- Economica, p. 49-58.

VANIER M. (2002). - « Les espaces du politique : trois réflexions pour sortir des limites du territoire ». In : DEBARBIEUX B., VANIER M., dir., Les territorialités qui se dessinent, Datar / Editions de l'aube, p. 75-89.

WACHTER S. et al. (2000). - Repenser le territoire. Un dictionnaire critique, Datar / Editions de l'aube, $288 \mathrm{p}$. 


\section{NOTES}

1. Consacrée par le rapport Brundtland en 1987, la notion de développement durable a été depuis largement adoptée par les responsables politiques et les techniciens de l'aménagement du territoire.

2. Selon l'INSEE, l'aire urbaine est un ensemble de communes d'un seul tenant et sans enclave, constitué d'un pôle urbain et de sa couronne périurbaine. Le pôle urbain est une agglomération qui offre au moins 5000 emplois. Quant à la couronne périurbaine, il s'agit des communes dont au moins $40 \%$ des actifs vont travailler dans le pôle.

3. Le périmètre d'attraction de l'agglomération strasbourgeoise a été défini sur la base de l'analyse des déplacements domicile-travail. Deux conditions ont été fixées pour qu'une commune soit considérée comme "agglomérée" à un pôle (entité d'une ou plusieurs communes) : le nombre des actifs allant travailler dans le pôle doit représenter à la fois plus de la moitié des actifs émigrant de la commune et dépasser l'effectif des actifs résidant et travaillant dans celle-ci (ADEUS, 1995). Le périmètre ainsi défini rejoint, à peu de chose près, celui de l'aire urbaine de l'INSEE.

4. Du temps de l'élaboration du nouveau périmètre du SD de la région de Strasbourg, la relance des politiques intercommunales était encadrée par la loi d'orientation sur l'organisation territoriale de la République du 6 février 1992.

5. Ceci a notamment été le cas avec les communes situées au nord-est de la CUS (Offendorf, Herrlisheim et Drusenheim), intégrées dans l'aire d'attraction du pôle strasbourgeois, mais faisant déjà partie du SD de la Bande Rhénane Nord (ADEUS, 1995).

6. En 1990, près de $24 \%$ de la population active de Molsheim ayant un emploi travaillait dans la CUS.

7. Collectivité territoriale qui possède des compétences en matière de développement économique, de transports collectifs interurbains, etc. et exerce de fait le rôle d'une structure intercommunale.

8. Respectivement Syndicat intercommunal à vocation unique et Syndicat intercommunal à vocation multiple.

9. Une des particularités de la CUS est d'avoir très tôt fusionnée l'administration municipale de Strasbourg avec l'administration communautaire.

10. Organe qui administre le Syndicat Mixte. Sur les 50 membres titulaires, 25 appartiennent à la CUS, le reste représentant les autres collectivités. C'est le président de la CUS qui est actuellement aussi président du Syndicat Mixte (élu par le Comité syndical).

11. Accord de Karlsruhe du 23 janvier 1996.

12. Le Syndicat Mixte est une structure de mise en place et de suivi du SCOT. Elle n'est pas une structure de mise en œuvre des projets retenus.

13. Entretien avec Christian Meyer, ADEUS, 27 septembre 2004.

14. Syndicat Mixte du SCOTERS, Compte rendu du bureau du 21 avril 2004.

15. La loi de la variété requise définie par le cybernéticien Ross Ashby stipule qu'un «système physique très riche en possibilités ne peut être contrôlé par un système de gestion pauvre et simpliste " (Mélèse, 1968, p. 65). Si l'on suit cette idée, face à la complexité des territorialités contemporaines, seule la complexité des territoires institués peut constituer une réponse adéquate. 


\section{RÉSUMÉS}

Dans un contexte de recomposition territoriale marqué par la montée en puissance de l'intercommunalité et la multiplication des territoires de projets, l'établissement d'un périmètre de SCOT constitue un moment privilégié pour analyser les dynamiques en jeu. Dans la région de Strasbourg, la mise en place de ce nouvel outil de planification éclaire les stratégies territoriales des acteurs locaux et les enjeux auxquels ils font face. Bien que liées par un destin partagé, la communauté urbaine et les intercommunalités périphériques poursuivent des stratégies divergentes dans le cadre des recompositions territoriales à l'œuvre. Le recouvrement partiel des différents territoires de projets et l'enchevêtrement des limites qui en résulte posent la question de l'articulation des périmètres et de leur cohérence.

In the context of territorial recomposition marked by the rise to power of inter-communality and the multiplication of projects' territories, the establishment of a SCOT perimeter provides an ideal moment for analysing the dynamics at work. In the Strasbourg region, the implementation of this new planning tool clarifies the territorial strategies of the local stakeholders and the issues which they face. Although bound by a shared destiny, the metropolitan authority and the peripheral districts pursue divergent strategies for territorial recomposition. The partial recovery of the various projects' territories and the entanglement of resulting boundaries poses the question of the articulation of the perimeters and their coherence.

Im Rahmen einer territorialen Umstrukturierung durch den Anstieg des Einflusses der Gemeindeverbände und die Vervielfachung der Projektterritorien stellt die Einrichtung eines SCOT einen bevorzugten Zeitpunkt dar, um die Dynamik in einem Gemeindegebiet zu analysieren. In der Region Straßburg beleuchtet die Einführung dieses neuen Planungswerkzeuges die territorialen Strategien der lokalen Handelnden und die Aufgaben, die zu bewältigen sind. Gebunden durch ein gemeinsames Schicksal verfolgen die Stadtgemeinde und die peripheren Gemeindeverbände unterschiedliche Strategien während der territorialen Umstrukturierung. Die partielle Abdeckung der verschiedenen Projektterritorien und die Verwicklung der Grenzen die sich daraus ergibt, stellen die Frage nach Konnexion der Umkreise und ihrer Kohärenz.

\section{INDEX}

Schlüsselwörter : Akteur, Planung, SCOT, Straßburg, Strategie, Territorium, Umstrukturierung Keywords : planification, recomposition, SCOT, stakeholder, Strasbourg, strategy, territory

Mots-clés : acteur, planification, recomposition, SCOT, Strasbourg, stratégie, territoire

\section{AUTEUR}

\section{CHRISTOPHE SOHN}

Docteur en géographie, Chercheur au centre d'études CEPS/INSTEAD, 44 rue Émile-Mark, B.P. 48, L-4620 Differdange, Luxembourg 